\title{
Rhetoric and reality of daily life in English care homes: the role of organised activities
}

\author{
By INGRID EYERS ${ }^{1}$, SARA ARBER$^{2}$, REBEKAH LUFF ${ }^{3}$, EMMA YOUNG $^{4} \mathcal{E}$ \\ THERESA ELLMERS ${ }^{2}$
}

\begin{abstract}
In divergent ways, both government policy and care home practices influence the everyday life of older people living in English care homes. The rhetoric of choice for care home residents may be in conflict with the reality of government policy-driven service delivery. The aim of the article is to examine the role of organised activities in facilitating choice and active ageing among care home residents. Findings from a study of ten care homes in South East England exemplify the conflict between government policy rhetoric and the reality of care home life. The indication is that the formality of the "activities of daily living" support procedures restricts residents' involvement in the organised social activities. Within the general provision of services, the organised "social activities" offered failed to meet the interest, cognitive and physical abilities of residents. The reality

\footnotetext{
${ }^{1}$ Ingrid Eyers, Zentrum Altern und Gesellschaft, University of Vechta, Vechta, Germany Guildford, UK

${ }^{3}$ Rebekah Luff, School of Social Sciences, University of Southampton, Southampton, UK
} ${ }^{2}$ Sara Arber, Theresa Ellmers, Centre for Research on Ageing and Gender, University of Surrey,

${ }^{4}$ Emma Young, Acute Stroke \& Brain Injury Unit, St. Peter's Hospital, Surrey, UK
\end{abstract}


International Journal of Ageing and Later Life

of "choice" is therefore questionable. Policy needs to support a transformation in the delivery of care to ensure it addresses the actual needs and expectations of older people experiencing care home life.

Keywords: activities of daily living, organised social activities, daily life, choice, care homes, policies, rhetoric and reality.

\section{Introduction}

Both government policy and care home staff practices influence the everyday life of older people living in English care homes, but these present divergent pictures. The rhetoric of "choice", "autonomy" and "dignity" for older people may be in conflict with the reality of policydriven service delivery in care homes. Rhetoric places the older person in the centre, yet in reality service providers aim to meet formulaic criteria set by governing/regulating bodies and commissioning purchasers.

Whilst the move into a care home is ultimately determined by an individual's health and social care needs, there is a growing awareness that there is more to care home life than providing assistance in personal care, ensuring medication is taken and nutrition is provided. Current government policy rhetoric in the United Kingdom (UK) is focused on facilitating empowered personalised care that is negotiated between resident and care staff (Morton \& Morgan 2009) and tailored to an individual's personcentred needs (Innes et al. 2006). The aim of this article is to examine the role of organised activities in facilitating choice and active ageing among care home residents, and in so doing address the relationship between policy rhetoric and reality in practice.

The study was undertaken in ten care homes in South East England. Evaluating the mundane, taken for granted, routine of daily life experienced by older people living in a care home provides insight into how physical and psychosocial aspects of residents needs are catered for.

\section{Background}

For the oldest old living in care homes a considerable step towards disengagement from society can be seen to have taken place; active ageing appears to take low priority and social capital diminishes. It is the need 
for support in the undertaking of formally identified "Activities of Daily Living" (ADLs) that has instigated the transformation in an older person's existence, resulting in their move into a care home. Ultimately, meeting care needs appear to become key determinants of care home residents' quality of life.

Resident's everyday life is determined by the need to adapt to community living (Eyers 2007), and detachment from both their previous living environment and social context becomes unavoidable. This reformed lifestyle within care homes is strongly regulated by UK government policy aiming to ensure basic outcomes are achieved (Care Quality Commission 2009; DH 2003) and influenced by a workforce aiming to provide care and support ADLs to the best of their ability (Eyers \& Bryan 2006). Within this revised lifestyle and new environment, the aim should be to maximise an individual's well-being and enable re-engagement in leisure activities previously enjoyed. In turn this should result in an overall improvement in the daily life experiences of older people living in care homes. The following section considers active ageing and the life of older people living in the community in contrast to that of care home residents.

\section{Active Daily Life in the Community and Care Homes}

Over the last ten years, active ageing as described by the World Health Organisation (Kaleche et al. 2005; WHO 2002) has been seen to be the panacea of old age. It is predicated on older people having the physical and cognitive abilities to actively and safely take part in society. In turn, society is required to accept and facilitate an environment that welcomes and includes older people. Everyday life for many autonomous old people reflects their personal life course (Clarke \& Warren 2007) and consists of a blend of activities ranging from sleeping at night to the pursuit of leisure activities and socialising with other people (Arber et al. 2003; Davidson et al. 2003; Gunnarson 2009). A further important factor is that activities have been found to have a positive impact on health (Agahi \& Parker 2005), well-being and self-esteem (Litwin \& Shiovtz-Ezra 2006).

Whether an older person lives at home or in a care home, the core principles of active ageing remain the same. However, as Johnson et al. (2010) identified in their comparison of present care home life with Peter 
International Journal of Ageing and Later Life

Townsend's (1962) classic study: "In both periods there were residents who were undermined by institutional living, and unhappy at the boredom and inaction imposed by the regime of some homes, and the lack of meaningful occupation" (Johnson et al. 2010: 139). Indeed in some instances Johnson and colleagues observed how a decrease in meaningful activities was now taking place because the residents no longer needed to earn money or help with housework, such as laying the table. As pointed out by one of the managers in the study by Johnson and colleagues, health and safety regulations limit such activities. Contrary to government rhetoric relating to autonomy and person-centred care, such findings imply a restriction in everyday activities. Before considering everyday life in care homes it is salient to initially consider how older people living "at home" fill their days and maintain their lifestyle.

Studies undertaken of the Swedish community-dwelling older people, aged over 75 (Gunnarson 2009; Paillard-Borg et al. 2009), identify reading as the main leisure activity closely followed by "making handicrafts". Mental activities (doing crosswords, cultivating political and cultural interests) were found to be very popular as were "productive activities" (gardening, handicrafts). Some of the least frequently undertaken activities among the oldest old included singing and playing bingo. The findings of Paillard-Borg et al. (2009: 809) provide a highly relevant insight into the reality of activities in later life. They list 31 activities, ranging from reading as the most frequent, down to reminiscing as the least frequent, and the findings exemplify how advanced age impacts on participation in leisure activities.

Within care homes, once support with the ADLs has been provided in the limited available time of approximately 29 minutes per resident (Eyers 2007), there is potentially sufficient time in the day for a care home resident to pursue individual activities and interests that present a continuation of their previous lifestyle. Such aspects of everyday life are relevant to empowered personalised care (Morton \& Morgan 2009) aiming to encompass choice, autonomy and dignity.

To date there has been little research on the lifestyle or leisure activities of older people living in care homes. If leisure activities are addressed they tend to be camouflaged by the term "rehabilitation" 
(e.g. Reilly et al. 2006), which in the UK links to National Health Service policies relating to the health of older people (DH 2001).

\section{Activities of Daily Living Within Care Homes}

The emphasis in care home research to date has predominantly been a reductionist approach to care giving (Lhussier et al. 2005; Markle-Ried \& Browne 2003), where care giving has been reduced to basic care with a focus on supporting ADLs. Within the context of both health and social care assessments, ADLs relate predominantly to personal hygiene, dressing and eating (Katz et al. 1963; Lawton \& Brody 1969; Roper et al. 2000). The formal conceptualisation of ADLs has provided the basis of care plans put in place for older people both in hospital and in care homes. The research literature relating to ADLs tends not to focus on leisure activities but only on an older person's ability to competently complete personal care and instrumental tasks to enable daily living (Warner Schaie et al. 2005). The framework of ADLs is geared towards ensuring that the relevant support or action by care staff is taken, so that a resident is washed, dressed, eats meals and is "toileted".

In the completion of these ADL tasks there is a constant conflict between "clock time" and "process time" (Eyers 2007). Whilst a member of staff might have five minutes to assist a resident with their breakfast, it might well take ten minutes for that person to eat their toast and drink a cup of tea. In addition, it might unexpectedly be necessary to wash the residents' face again and change their clothes. In this instance, care staff are likely to change the clothes without questioning which clothes the resident might like to wear (Eyers 2007).

In reality, care staff have little time to accommodate choice, autonomy or individuality; they have a job to do and according to both LeeTreweek (1997) and Ungerson (2000), a "product to produce". Supporting and undertaking ADLs are vital to the daily well-being of care home residents and central to the work undertaken by care staff. Indeed, the inability of an older person to undertake these tasks independently is closely linked to the reason they now live in a care home. However, given the available direct contact time between staff and residents to support the 
International Journal of Ageing and Later Life

ADLs, facilitating the complex care needs of a group of residents may well conflict with preferred "social activities" of an individual resident.

\section{Policy Frameworks Regarding Daily Care Home Life}

Research has shown that leisure and social activities have a strong relationship with well-being in later life (Havighurst \& Albrecht 1953; Rowe \& Kahn 1997). It is therefore important to evaluate the 24-hour day experienced in care homes and to consider the interconnection between staff undertaking ADLs and residents' opportunities to engage in "social activities". At the time of data collection for this article (20072008) the UK National Minimum Standards for Care Homes for Older People (DH 2003) regulated the registration of service providers in the care home sector. This policy document is relevant to service regulators, care home providers and service users, and indicates the expected minimal standard of achievements required in order to obtain and maintain registration to operate the care home. The overall aims of the Standards acknowledge the "... unique and complex needs of individuals ..." and aim to "... deliver an individually tailored and comprehensive service ..." (DH 2003: vii).

Within Chapter 3 of the document, daily life and social activities are outlined under Standards 12-15. Here choice, variety, opportunity and flexibilities are specified as key characteristics expected of the care home service provided. Highlighted text in the document states: "Service users are helped to exercise choice and control over their lives" (DH 2003: 15). The aims of the Standards and especially the text in Chapter 3 indicate that policy makers are very much aware of the importance of daily life activities. The successful completion of instrumental ADLs such as washing and dressing in the morning prepares an older person for the rest of the day and is an important step towards residents being able to actively participate in care home life.

At the time of data collection, health and social care policies in England were under review and at government policy level, the "Berlin Wall" (Glendenning et al. 2002) between health and social care services was being prepared for deconstruction. Wide ranging discussions were taking place and a White Paper titled "Our health, our care, our say: 
A new direction for community services" (DH 2006) was published. The document "Putting People First: A shared vision and commitment to the transformation of Adult Social Care" exemplified how the National Health Service and policies on Adult Social Care should work together (DH 2007). An explicit theme was personalised care to ensure the best possible quality of life through "enablement and high quality personally tailored services... maximum choice, control and power ..." (DH 2007: 2). Within English government policy documents and information brochures, promoting dignity was a key concept, irrespective of age. It was, however, also discreetly stated that "the right to self-determination will be at the heart of a reform system only constrained by the realities of finite resources and levels of protection which would be finite but not risk averse" (DH 2007: 2). Thus, indicating an acknowledgement that there may be a conflict between rhetoric and reality.

Since completion of our data collection, the regulation of care home services has transferred from the UK Commission for Social Care Inspection (CSCI) to the Care Quality Commission (CQC) established in April 2009. All care homes in England are now required to register with the governing body that regulates the provision of care home services. The role of the CQC ranges from regulating the architecture of the building, to the quantity and quality of staff, and to the everyday activities provided for residents. A review of the guidelines has been undertaken, and under current policy (November 2011) respecting and involving people who are being provided with a service is identified as an important outcome and can be linked to everyday activities (CQC 2009).

Within a case study used by CQC as an example of bad practice, reference is made to the inability of people with learning disabilities living in a care home to attend a pub quiz because it takes place at a time when a change of staff takes place (CQC 2009: 41). A further CQC example of bad practice relates to the independence of older people living in a care home. It describes how residents were required to use a wheelchair, although they were able to walk with the assistance of a walking frame, because it saved staff time and prevented falls (CQC 2009: 40). These examples of everyday life in care homes, used to exemplify recognised bad practice and assessment outcomes, were seen, respectively, to be of major and moderate concern. As such they indicate the policy interpretation of 
International Journal of Ageing and Later Life

personalised care. These more measurable aspects of care contrast with the less tangible characteristics of personalised care, which focus on the ability of care staff to have patience, be compassionate, sensitive and be able to empathise with a care recipient whilst supporting them to perform ADLs.

\section{Research Design}

The aim of this study was to better understand everyday life in care homes by focusing on the role of organised leisure activities, and how these are influenced by the staff practices to accomplish ADLs for residents. In ten purposively selected care homes for older people situated in South East England, quantitative and qualitative data were collected from 145 male and female residents, aged 60-100 years. In order to ensure a high number of participants were able to give informed consent, only care homes registered for $35+$ residents were selected. The ownership of the homes covered local authorities, the voluntary sector, large corporate organisations and small businesses (see Table 1). The intention was to capture the breadth of service providers encountered in the UK where in the norm care homes are independent of the National Health Service. Ownership encompasses municipal Social Services, the voluntary sector, small family businesses and large corporate organisations. For many years, the majority of care homes operated in buildings that were formally large family homes or mansions that were converted into care homes with extensions added. At the time of data collection, a phase of transition was taking place, as many of the converted buildings no longer met the minimum building

Table 1. Characteristics of ten studied care homes

\begin{tabular}{|c|c|c|c|c|c|c|c|c|c|c|}
\hline Selection criteria & 1 & 2 & 3 & 4 & 5 & 6 & 7 & 8 & 9 & 10 \\
\hline No. of beds & 60 & 44 & 38 & 38 & 72 & 43 & 58 & 74 & 82 & 47 \\
\hline Vol. sector owner & $\checkmark$ & & & & & & & & & \\
\hline Local authority owner & & $\sqrt{ }^{\mathrm{a}}$ & $\sqrt{ }^{\mathrm{a}}$ & $\sqrt{ }^{\mathrm{a}}$ & & & & & & \\
\hline Corporate owner & & & & & $\sqrt{ }^{\mathrm{b}}$ & $\sqrt{ }^{\mathrm{b}}$ & $\sqrt{ }^{\mathrm{b}}$ & $\checkmark$ & $\sqrt{ }$ & \\
\hline Small business & & & & & & & & & & $\checkmark$ \\
\hline Purpose build & $\checkmark$ & $\checkmark$ & $\checkmark$ & $\checkmark$ & $\checkmark$ & $\checkmark$ & $\checkmark$ & $\checkmark$ & $\checkmark$ & \\
\hline Conversion & & & & & & & & $\checkmark$ & $\checkmark$ & $\checkmark$ \\
\hline
\end{tabular}

\footnotetext{
${ }^{\mathrm{a}, \mathrm{b}}$ Same ownership.
} 
standards set out in the National Minimum Standards for Care Homes for Older People (DH 2003). Geographically the homes were situated in suburban and rural areas and aimed to cover a spread of socio-economic groups. All the participating facilities met the required National Minimum Standards for Care Homes for Older People (DH 2003) and consequently were considered to fulfil the statutory criteria set by policy makers.

Prior to conducting the research, information about the study was provided to the home management, all care home staff, residents and their relatives. On the day that the data collection commenced, a presentation was made to residents and staff in each home. Residents who had volunteered and were cognitively able to participate in the study each had a detailed conversation with the researchers before signing the consent form to participate in the study, which included keeping a daily diary $\log$ over a period of two weeks denoting their everyday activities. As the majority of participants were not able to personally maintain the diaries, researchers visited daily to record the details of the previous 24 hours. This was done in questionnaire format, which standardised the data collected and facilitated the analysis using SPSS. The aim of the diaries was to gain an insight into resident's everyday life. Each diary also recorded demographic information, and staff provided an assessment of the participants' dependency level based on the amount of time needed to provide care every day (Eyers 2000; 2007). Audio-tape recorded interviews were conducted with all ten care home Managers and with the Activities Organisers in each home. Insight into the support required with ADLs was based on the participating residents' dependency level and continence care needs. Further information regarding the overall ADLs met within each care home was provided by the matron/manager within their interview.

Whilst the diary data was recorded in questionnaire format, the researchers actually conducted conversations with residents to elicit the relevant data. These conversations were held in an informal way and resulted in a range of topics being covered, contributing to the richness of the data.

Daily observational studies were undertaken over a two-week period in each of the care homes. On average, at least two hours were spent in each home at varying times of day, including dawn, dusk and over-night, 
International Journal of Ageing and Later Life

resulting in a total of 250 hours direct observation. Field notes were maintained in conjunction with the completion of the diaries, which took at least a further two hours per day. The focus was on the activities taking place and the researcher was able to participate in and observe the implementation of the organised activities programme provided in each home. Documentary data in the form of programmes and newsletters relating to the organised activities were also collected. Undertaking the observations and the daily visits to maintain the diaries meant that the key researcher spent a considerable amount of time in each home and was able to assess the validity of the information provided by the participating residents.

This multi-method approach facilitated "rigor without rigidity" (Fielding 2009: 428) for this study with vulnerable older people living in a complex community. Ethical approval for the study was given by the University Ethics Committee and Local Authority Social Research Governance. The names used in this article are pseudonyms to preserve confidentiality.

\section{Characteristics of the Participating Care Home Residents}

Diary data was collected from 145 residents who completed all or part of the data sets. There were 105 women and 40 men, covering an age range of 60-100 years, with the strongest representation of both genders in the age group of 85-89 years.

Information about the participant's health care needs was provided by care staff and indicated that 27 did not require physical assistance, whilst 79 received 90-180 minutes of physical care and support per day. Three to five hours of "hands-on" care was provided to 30 residents, whilst three participants received over five hours of assistance to meet their health care needs. Data relating to dependency were missing from three participants. As the background data collected about the care homes provided by matron/managers from five care homes indicated, assisting and supporting residents to use the toilet was, alongside assistance in getting washed and dressed, one of the main staff-assisted ADLs of the day (see Figure 1). 
Figure 1. Percentage of residents requiring support with ADLs (matron/ manager data from 5 participating care homes)

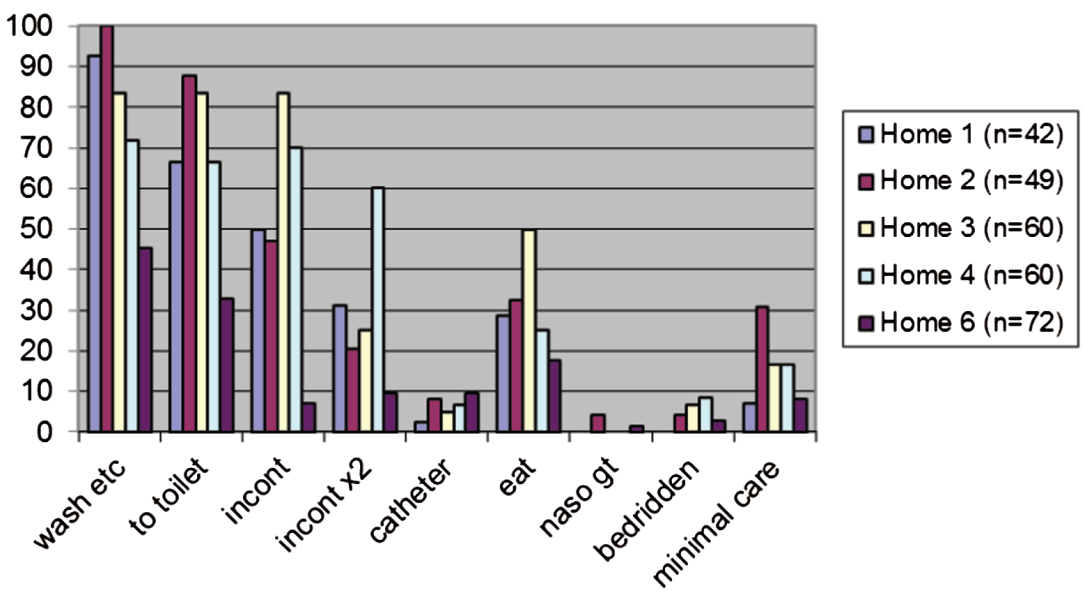

Wash etc: assistance with washing, getting out of bed, dressing; To toilet: assistance to use the toilet; Incont: dealing with residents who are incontinent of urine and wear a pad or special underwear; Incont $x 2$ : dealing with residents who are incontinent of urine and faeces and wear a pad or special underwear; Catheter: residents with a urine catheter inserted in the bladder; Eat: residents who need assistance to eat a meal; Naso gt: nasogatric tube (artificial feeding); Bedridden: residents who do not get out of bed; Minimal care: residents who get washed and dressed independently but for example need supervision to take medication and eat.

The data from the five homes in Figure 1 were typical of all ten care homes. As the following section exemplifies, providing support in the above-described activities determines the rhythm of the day for both residents and care staff. Care staff were constantly aware of their limited time resources (see also Eyers 2007), and residents had plenty of time to undertake leisure activities. 
International Journal of Ageing and Later Life

\section{Supporting ADLs: The Rhythm of the Day}

The rhythm of care home life is determined by the interaction between residents and staff providing support with ADLs. The morning in a care home starts early and is heralded by the onset of activities related to "continence checks" (approx. 4 a.m.) followed by the clatter of the tea trolley and the early morning "drugs round". The day draws to an end with the serving of the evening meal (supper) usually at $5.30 \mathrm{pm}$, and by $7 \mathrm{pm}$ most residents are in their individual rooms and often in bed by $8 \mathrm{pm}$. As identified by Luff et al. (2011), the mean time care home residents spend in bed is 10 hours 50 minutes. For residents needing the assistance of a hoist to be lifted, their personal bed time is determined by the availability of two members of staff to undertake this task. As one participant who needed to be "hoisted" pointed out, she did not really want to go to bed early but she appreciated the fact that due to the lower staffing levels after 8 pm she had no other choice.

Residents were seen to change previous everyday habits and try to "adapt" to the care home as a form of communal living. This is exemplified by a comment made in an interview with one of the matrons who said:

... I notice when I go out to assess people in their own homes they say "I stay up until 11 o'clock and I watch TV in bed". But within a very short period of time, they are going to bed at 9 o'clock. (Joan, Care Home Manager)

This interview extract also indicates how the institution influences the individual and is underpinned by the following field note observation.

\footnotetext{
... At approximately 7 p.m., when nearly all the other residents were in their rooms, a new resident was offered the choice to stay in the conservatory or to go upstairs to her room. Choice was offered verbally in a form that also made it clear that the member of staff considered it preferable for the new resident to go to her bedroom. Although the new resident vocalised that it was too early to be going to bed, she realised that there was nothing to stay downstairs for, no fellow residents to chat with, no activities, therefore going to her room was the only option and she took it. Her only choice at $7 \mathrm{pm}$ was between being isolated in the conservatory or in her bedroom. The day shift was coming to an end and care staff needed to be seen to have prepared the home for the night staff. All the other residents were in their rooms and, if not already in bed, at least ready to go to bed.
} 
These data extracts exemplify how choice and autonomy are limited within the confines of a care home and life style changes become unavoidable.

Based on data from one home (where care staff worked in 12-hour shifts) it was established that during the day $(8 \mathrm{am}-8 \mathrm{pm})$, the ratio of staff (13) to residents (49) meant that within the 12-hour shift period, 2.9 hours contact time was available to support and assist each resident with their ADLs. This leaves the remaining 9.1 hours in the hands of the individual resident or the Activities Organiser or to fill the day with active or passive leisure and recreational activities. The rhythm of the institutional day appears to dominate over a personal daily routine, which has previously been embedded in the life course of the oldest old who are now living in a care home.

Little opportunity appeared to remain for resident choice or individuality, which could theoretically be related to person-centred care or personalised care. The care home day resembled that of the clinical model encountered in a hospital rather than the lifestyle of an older person living in their accustomed personal home environment. The policy rhetoric related to individual choice, autonomy and dignity is confronted by the reality of institutional care home life. In the following section, an overview of the formal organised activities offered in the care homes is provided and their relationship to ADLs is considered.

\section{Activities of Daily Living versus Formally Organised Activities}

The nature of this study meant that the participants were amongst the most active in each of the ten care homes. The following analysis of "formally organised activities" provides evidence of a hospitalised lifestyle in care homes that has little resemblance to that of the residents' contemporaries living in the community.

From the two-week diary data relating to activities, which was completed by 125 of the participants, it was found that participation in organised activities was low with $51 \%$ of residents not participating in any morning activities and $42 \%$ not participating at all in the afternoon across the two-week period (see Table 2). The $2 \%$ who did participate in an evening activity reflects participants who attended the one evening activity 
International Journal of Ageing and Later Life

Table 2. Diary data indicating the average number of activities attended per week (in percentage)

\begin{tabular}{lcc}
\hline Time of activity & None $(0.5<2)$ & 2 or more (total) \\
\hline Morning & $51(25)$ & $24(n=125)$ \\
Afternoon & $42(35)$ & $23(n=125)$ \\
Evening & $98(2)$ & $0(n=125)$ \\
All organised activities & $29(31)$ & $40(n=125)$ \\
\hline
\end{tabular}

that took place in one home during the two-week data collection period. Overall, 29\% of residents did not participate in any organised activities across the two-week study period, and only a minority of residents $(40 \%)$ participated in two or more activities per week. Since the organised activities usually lasted no more than $1-1 \frac{1}{2}$ hours, this indicates that care home residents spent only a tiny amount of their time involved in organised leisure activities.

The field notes and interview data indicated that residents had limited actual interest in the organised activities provided. An indication that this could be related to the timing of activities was observed when morning activities were delayed to 11.00 a.m. This later starting time was observed to result in an increased number of residents participating. This may also be a factor in the slightly higher participation in afternoon activities, as shown in Table 2. Participation in the organised activities for many is dependent on care staff taking them to the relevant room in the home, and observational data suggested that much of the morning is taken up by the completion of formally acknowledged ADLs. The timing of organised activities was not always suited to care staff's ability to prepare residents in time to attend. At this point, the completion of ADLs, representing the identified care needs that initiated the move into a care home, conflicts with "social activities" described within the National Minimal Standards for Care Homes (DH 2003). As previously stated, Standards 12-15 do place an emphasis on leisure activities and structured provision of a social activities programme in care homes.

The organised social activities encountered in the ten care homes were primarily related to music, arts and crafts, with occasional outings to local parks or gardens. Despite the range of homes (see Table 1) participating in 
the study, there was ultimately little difference in content of the organised activities programme in the participating homes. Very few of the observed activities could be categorised as "rehabilitation", which would for example encourage a resident who had suffered a "Stroke" to regain dexterity or joint mobility without receiving dedicated Physiotherapy. On the other hand, the observational data and informal conversations with residents indicated that they perceived many of the arranged activities to be bland, almost childish and often inappropriate. The extract from one of the care homes' activities programme for a typical week (Table 3) shows that music in the form of listening to music, alongside reading the newspaper, exemplifies the passive (non-physical) activities organised within the care homes.

Whilst "baby talk" was not observed in communication between residents and staff, a form of institutional infantilisation in terms of the content of the organised activities was taking place and indicates that a loss of dignity and respect was occurring. In one home, especially when entering the building, you could be excused for thinking you were entering a nursery school building with childlike art and craft work on display. Whilst it could be argued that it is good to see that organised activities are taking place, for some older people and their relatives it must be demeaning to see yourself or your parent publicly being reduced to childlike activities.

By focusing on organised activities relating to music, seen as a fundamental component of human life (Biley 2000), it becomes clear that an activity, which may initially appear suitable to management, Activities Organisers and the care home inspectorate, was not always provided in a format welcomed by older care home residents. Choice and individual interests that would reflect a person-centred or personalised care approach failed to be a component of service delivery. However, the service offered could be "formally" seen to be in keeping with the National Minimal Standards for Care Homes (DH 2003).

The following field note extracts exemplify a conflict between policy rhetoric and actual care provision indicating a lack of knowledge and awareness of residents' previous lifestyles, current interests or abilities. In dialogue with residents who were maintaining a diary with the support of the researcher, the following comments relating to musical activities were 
International Journal of Ageing and Later Life

Table 3. Extracts from an activities programme in one typical care home

\begin{tabular}{|c|c|c|}
\hline Monday & 11.00 am: Shoulder Class & $2.00 \mathrm{pm}$ : Annie with Freddie \\
\hline Hairdresser & 20 mins exercise & and Ginger (Pat dogs) \\
\hline All day & Ground Floor Lounge & $\begin{array}{l}3.00 \text { pm: Tea Rooms and } \\
\text { Home Shop } \\
\text { Ground Floor Lounge }\end{array}$ \\
\hline Tuesday & $10.30 \mathrm{am}$ : Coffee and home-made & 2.30 pm: Home Choir with \\
\hline Hairdresser & cakes. Bring your newspaper. All & Billy Songster \\
\hline All day & $\begin{array}{l}\text { welcome } \\
\text { Ground Floor Lounge }\end{array}$ & Ground Floor Lounge \\
\hline Wednesday & $\begin{array}{l}\mathbf{1 0 . 3 0} \text { am: Coffee and home-made } \\
\text { cakes. Bring your newspaper. All } \\
\text { welcome } \\
\text { Ground Floor Lounge }\end{array}$ & $\begin{array}{l}2.30 \text { pm: Exercise Classes } \\
\text { Ground Floor Lounge }\end{array}$ \\
\hline Thursday & $\begin{array}{l}\mathbf{1 0 . 3 0} \text { am: Coffee and home-made } \\
\text { cakes. Bring your newspaper. All } \\
\text { welcome } \\
\text { Ground Floor Lounge }\end{array}$ & $\begin{array}{l}2.30 \text { pm: Beauty Nail Care } \\
\text { with Sherry and Relaxing } \\
\text { Music } \\
\text { Ground Floor Lounge }\end{array}$ \\
\hline \multirow[t]{2}{*}{ Friday } & $\begin{array}{l}\mathbf{1 0 . 3 0} \text { am: Sherry with Relaxing Music } \\
\text { Lounge Ground Floor } \\
\mathbf{1 1 . 0 0} \text { am: Holy Communion } \\
\text { Lounge Ground Floor }\end{array}$ & $\begin{array}{l}2.30 \text { pm: Bingo } \\
\text { Ground Floor Lounge }\end{array}$ \\
\hline & Lunch: 12.30 pm & Supper: 5.30 pm \\
\hline
\end{tabular}

made: "I have no inclination to sing the Lambeth Walk, thank you very much". This was said by Emily who loved classical music and often listened to it in her room and found "the man" who arranged and accompanied the choir offensive, as by her standards he did not play the piano adequately.

Field notes from another home exemplify a situation where residents did express discontent:

... A man, who couldn't sing, was singing at the residents. He failed to interact with the people he was entertaining. No eye contact was made and there was a distinct lack of dialogue with the residents. However, some residents were reacting by yelling "shut up" and "get off". Others who had been wheeled in by the staff, sat there motionless. 
These extracts from the field notes exemplify that some residents do have strong opinions about the organised activities. Residents were clearly vocalising discontent, but this was not being heard, indicating that choice, variety, opportunity and flexibility had little space within the activities programme.

The interviews with the Activities Organisers indicated that they were oblivious to residents' opinions about the activities, and that they were confident they were providing suitable activities to the best of their ability. Our data emphasise that taking a generalised simplistic approach to providing organised "social activities" is not appropriate and needs to be reconsidered. However, many of our research participants did not want to be seen to make a fuss and appreciated that the existing Activities Programmes were well intended. This implies that the residents were acutely aware of the situation that care staff were confronted with daily and further indicates the adaptation process an old person undertakes after the transition into care home life has taken place. This also reiterates the importance of identifying and acknowledging each resident's interests and abilities as part of a person-centred service delivery that takes both body and soul into consideration.

\section{Accommodating Organised "Social Activities" Into the Routine of the Care Home}

During the data collection it was noted that programmed morning activities were more likely to be cancelled than those in the afternoon. The morning was the busiest time of the day, and the main aim of care staff appeared to be to ensure that, in accordance with the care plans, support with ADLs was provided. Activities offered in the afternoon tended to be slightly more popular with the residents, and evening activities only took place once in one home during the two-week study across the ten care homes. Only in the most exclusive of the care homes was it observed that residents independently arranged evening games of "Scrabble". Regular weekend activities were not the norm; however, all the participating homes did host "events" such as a Summer Fayre that took place during data collection in one home and was enjoyed by all who were able to attend and participate. The findings from the daily fieldwork 
International Journal of Ageing and Later Life

observations indicated that the working hours of Activities Organisers were not in tune with the resident's daily routine and consequently restricted residents' ability to participate if they were not washed and dressed in time to be in the relevant room at the start of an organised activity.

In all care homes, it was noticeable that when the person employed to facilitate "formal activities" was absent, they were not replaced, and formal activities did not occur. In some homes the Activities Organiser was employed on a part-time basis and was not in the care home each weekday. The general lack of activities after $4 \mathrm{pm}$ also indicates the driving role the Activities Organiser has in the "active ageing" experienced in care homes. Formal activities were not always intended to take place daily, and weekends or evenings were rarely taken into consideration as a time when it would be suitable or appropriate to facilitate organised "social activities".

\section{Linking ADLs to "Social Activities"}

Whilst there is clearly an issue relating to the appropriateness of the organised social activities, the data indicate that the "gatekeepers" to participation in activities were the care staff. Residents were dependent on care staff availability to wash, dress and assist them to the venue. The field notes also indicated there was a need for staff to remind residents every morning of the arranged activity for the day. Furthermore, care staff perceived that physical dependency was linked to participation, disregarding older people's intellect, interests and abilities.

The uptake of the activities provided was low, and there was an apparent disinterest in the provided programmes. The interview and diary data in conjunction with the field notes showed that overall it was mainly the same group of residents who participated. In many of the homes it appeared to be accepted by staff that many residents would not leave their room. However, many of the research participants who rejected the organised activities did enjoy social interaction as was evident through the conversations with the researchers held on a daily basis during the two-week data collection. 
When the Activities Organisers were asked what activities residents seemed to enjoy the most, they identified "outings" to be the most popular. From the dialogue between the researchers and residents, the popularity of trips outside the boundaries of the care home was also obvious. However, as was pointed out by the interviewed Activities Organisers and managers, this required the involvement of care staff, which unavoidably resulted in staffing problems as extra staff would need to be on duty. Cost and the requirement to undertake a risk assessment for each trip outside the home also needed to be taken into consideration. This invariably curtailed the amount of activities outside the care home environment.

Most Activities Organisers claimed to be willing to facilitate individual activities for residents on a one-to-one basis. However, this was actually only observed in one care home. When asked about individual activities the responses indicated that meeting the needs of all residents was difficult, underpinned by one interviewee who pointed out that she was "on her own doing her best to meet the requirements of over 35 residents". This comment reflects the conflict that care home staff encounters on a daily basis and underpins the complexity of addressing the gap between government rhetoric and care home reality.

\section{Discussion}

The organised activities observed in this care home study reflect those described by Goffman (1961) in his seminal work on institutional life. The "attempts by staff to manage the daily activity of a large number of persons in a restricted space with small expenditure of resources" (Goffman 1961: 50) were also observed in this study. In an institutional environment it is unavoidable that trying to please the majority takes precedence over the rhetoric of choice, and the pursuit of personal interests and respect for individuality are confronted by the reality of resources, formal structures and procedures. The principle objective of a care home is to provide a care service to a group of vulnerable older people, in a large building with communal areas. The policy rhetoric of person-centred care that facilitates choice, autonomy and dignity is put into question. 
International Journal of Ageing and Later Life

The indication is that in practice lip service is being paid especially to Standards 12 and 13 of the National Minimal Standards for Care Homes for Older People (DH 2003). In addition, presenting a "social activities programme" forms part of the marketing tools used to promote a care home to potential new customers. It also pacifies relatives, friends and the care home inspectorate. However, establishing their views on the organised activities provided was not part of this study and would call for further investigation.

For an older person living in a care home, mundane life appears to be based on a blend of care assistants' support with formally identified ADLs and the option to participate in an organised "activities programme" that seldom appears to be appropriate or attractive. The majority of programmes in the sampled homes indicate little knowledge or understanding of older peoples' actual interests either as individuals or as social groups. There is little opportunity for residents to pursue interests they have developed over their life course, nor is consideration given to how activities might impact on an individual's health, well-being or self-esteem. Despite all UK government rhetoric focusing on dignity, individuality and choice, the institutional requirements dominate over those of the individual.

The care home day, in conjunction with care home managers and staff, can be deconstructed to identify core elements that must be maintained. If a component such as the timing of meals were to be reconsidered to facilitate flexibility and choice, it could for example result in the construction of a more individualised daily timeframe for residents. Less prescriptive meal times provided in a restaurant style would for example mean that leisure activities could possibly be facilitated in a different, more personalised way, and would potentially facilitate the socialisation of like-minded people.

It is crucial that the importance of the Activities Organiser is acknowledged and formalised by policy makers to ensure skilled services are provided that enhance the lives of older people living in a care home. To ensure a truly holistic service provision that encompasses all the needs of an individual would be an alternative that meets Standards 12-15 (DH 2003) and also extends the role of care staff, by enabling them to 
facilitate all forms of everyday activities. The formalised ADL tasks related to getting a resident up, washed and dressed in the morning is after all the first and most important step towards enabling a resident to pursue or participate in activities that are important to their quality of life. The second step to then facilitate informal daily activities would be a natural progression. If this was the case, the daily routine experienced by older people living in a care home would be able to resemble more that of their contemporaries living in their own homes than that of a patient in hospital.

Enabling care staff to provide such a holistic service would invariably require relevant training and an increased ratio of staff to residents. Currently, residents have a surplus of time whilst staff has barely enough time to provide support and assistance with acknowledged ADLs. A third solution could be for structures to be put in place that would enable and encourage voluntary organisations to be actively involved in the daily social life in care homes as part of a reconstructed daily routine. The care homes studied during the research were providing the best possible services under the given circumstances, which are determined by how care homes are funded and regulated. Only if policy drives change in care homes will it become the norm that a balance is struck between meeting the needs of both body and soul.

\section{Conclusion}

For the oldest old living in care homes, the UK Department of Health (CQC 2009) rhetoric of dignity, choice, variety, opportunity and flexibilities, alongside a focus on the facilitation of person-centred care is far from the reality experienced. The reductionist ADLs of an older person living in a care home become a dominant feature. They relate to factors that can be visually assessed by both professionals and relatives, leaving little space for the less measurable, at times invisible and intangible, aspects that are important to the well-being of every human being's life, irrespective of age. At the simplest level, it is possible to see if someone is wearing clean clothes and smell that they have not been incontinent and have been washed. To measure or assess the outcome of social interaction or a leisure 
International Journal of Ageing and Later Life

activity, such as listening to music, is more complex and appears therefore to be overlooked.

In this research the conflict, between care staff supporting ADLs and residents participating in organised "social activities" within a care home became evident. We conclude that there is a need to deconstruct the standardised framework in which institutional care is provided and to reconstruct a frame that facilitates and respects individuality. A transformation in the philosophy of care needs to take place to enhance the everyday life encountered in care homes and to enable the oldest old to maintain a lifestyle to which each individual is accustomed and which is adapted to their physical and cognitive abilities. The rhetoric of choice, autonomy and dignity needs to realistically consider the importance of balancing residents' physical care needs with the less tangible aspects of mundane life.

A care home is not a clinical environment; therefore, it is inappropriate to reflect the routine of hospital life. Whilst the need to live in a care home is rooted in frailty or a medical condition resulting in the need for support in accomplishing ADLs, it is also of great importance that the formal activities taking place in care homes reflect the resident's intellect, interests, physical and cognitive abilities. This would bring body and soul in tune and actually result in a service that centres on the individual person. To ameliorate the current situation, future developments in both policy and practice need to give these factors of care home life due consideration.

\section{Acknowledgements}

The authors would like to thank the older people who allowed us into their everyday life in a care home and participated in this research. Our gratitude is also expressed to the ten care homes that enabled us to conduct the data collection on their properties. All the homes were providing care to the best of their ability. Points raised in this article aim to support them and help improve the lives of both residents and staff. Last but not least, many thanks to Karen Bryan, Cynthia Wyld, Robert Meadows and Wendy Knibb for their contributions and support in collecting this data during a difficult time for the original research team. The SomnIA research was supported by the New Dynamics of Ageing 
initiative, a multidisciplinary research programme funded by AHRC, BBSRC, EPSRC, ESRC and MRC (RES-339-25-0009). This is a revised version of a paper presented at the "Transformation of Care Conference" in Copenhagen in June 2010.

\section{Corresponding Author}

Ingrid Eyers, Zentrum Alter und Gesellschaft, University of Vechta, PO Box 1553, 49364 Vechta, Germany. Email: ingrid.eyers@uni-vechta.de

\section{References}

Agahi, N. \& Parker, M. (2005). Are today's older people more active than their predecessors? Participation in leisure-time activities in Sweden in 1992 and 2002. Ageing and Society 25(6): 925-941.

Arber, S., Price, D., Davidson, K. \& Perrin, K. (2003). Re-examining Gender and Marital Status: Material Wellbeing and Social Involvement. In S. Arber, K. Davidson \& J. Ginn (eds.), Gender and Ageing. Changing Roles and Relationships (pp. 148-167). Maidenhead: Open University Press.

Biley, F. C. (2000). The effect on patient well-being of music listening as a nursing intervention: A review of the literature. Journal of Clinical Nursing 9: 668-77.

Care Quality Commission (CQC). (2009). Guidance About Compliance: Judgment Framework. London: Care Quality Commission.

Clarke, A. \& Warren, L. (2007). Hopes, fears and expectations about the future: What do older people's stories tell us about active ageing? Ageing \& Society 27(4): 465-488.

Davidson, K., Daly, T. \& Arber, S. (2003). Exploring the Social Worlds of Older Men. In S. Arber, K. Davidson \& J. Ginn (eds.), Gender and Ageing. Changing Roles and Relationships (pp. 168-185). Maidenhead: Open University Press.

Department of Health (DH). (2001). National Service Framework for Older People. London: HMSO.

Department of Health (DH). (2003). Care Homes for Older People, National Minimum Standards. London: HMSO. 
International Journal of Ageing and Later Life

Department of Health (DH). (2006). Our Health, Our Care, Our Say: A New Direction for Community Services. London: Crown Copyright.

Department of Health (DH). (2007). Putting People First: A Shared Vision and Commitment to the Transformation of Adult Social Care. Available on http://www.dh.gov.uk/en/Publicationsandstatistics/Publications/ PublicationsPolicyAndGuidance/DH_081118 (Accessed: May 8, 2009)

Eyers, I. (2000). Education and training. Do they really, really want it? A comparative study of care home staff in England and Germany. Education and Ageing 15(2): 159-175.

Eyers, I. (2007). Extracting the essence of formal caregiving: A comparative study of formal care givers in English and German care homes. In I. Paoletti (ed.), Family Caregiving to Older Disabled People: Relational and Institutional Issues (pp. 273-294). New York, NY: Novascience.

Eyers, I. \& Bryan, K. (2006). Professionalizing the care of older people: Transforming the workforce. Quality in Ageing 7(4): 37-45.

Fielding, N. (2009). Going out on a limb: Postmodernism and multiple method research. Current Sociology 57(3): 427-447.

Glendenning, C., Coleman, A. \& Rummery, K. (2002). Partnerships, performance and primary care: Developing integrated services for older people in England. Ageing and Society 22(2): 185-208.

Goffman, E. (1961). Asylums: Essays on the Social Situation of Mental Patients and Other Inmates. New York, NY: Anchor.

Gunnarson, E. (2009). 'I think I have a good life': The everyday lives of older women and men from a lifecourse perspective. Ageing and Society 29(1): 33-48.

Havighurst, R. J. \& Albrecht, R. (1953). Older People. New York, NY: Longmam, Green and Company.

Innes, A., Macpherson, S. \& McCabe, L. (2006). Promoting Person-centred Care at the Front Line. York: Joseph Rowntree Foundation.

Johnson, J., Rolph, S. \& Smith, R. (2010). Residential Care Transformed: Revisiting 'The Last Refuge'. Basingstoke: Palgrave Macmillan.

Kaleche, A., Barreto, S. M. \& Keller, I. (2005). Global ageing: The demographic revolution in all cultures and societies. In M. Johnson, V. L. Bengtson, P. Coleman \& T. B. L. Kirkwood (eds.), The Cambridge Handbook of Age and Ageing (pp. 30-46). Cambridge: Cambridge University Press. 
Katz, S., Ford, A., Moskowitz, R., Jackson, B. \& Jaffee, M. (1963). Studies of illness in the aged: The Index of ADL, a standardized measure of biological and psychological function. JAMA 185: 94-99.

Lawton, M. P. \& Brody, E. M. (1969). Assessment of older people selfmaintaining and instrumental activities of daily living. Gerontologist 9: 179-185.

Lee-Treweek, G. (1997). Women, resistance and care: An ethnographic study of nursing auxiliary work. Work, Employment and Society 11(1): 47-63.

Lhussier, M., Watson, B., Reed, J. \& Clarke, C.L. (2005). The SEIQoL and functional status: How do they relate? Scandinavian Journal of Caring Sciences 19: 403-409.

Litwin, H. \& Shiovitz-Ezra, S. (2006). The association between activity and wellbeing in later life: What really matters? Ageing $\mathcal{E}$ Society 26(2): 225-242.

Luff, R., Ellmers, T., Eyers, I., Young, E. \& Arber, S. (2011). Time spent in bed at night by care homes residents. Ageing and Society 31(7): $1229-1250$

Markle-Ried, M. \& Browne, G. (2003). Conceptualization of frailty in relation to older adults. Journal of Advanced Nursing 44(1): 58-68.

Morton, T. \& Morgan, M. (2009). Examining how personalized care planning can help patients with long term conditions. Nursing times. net. Available on http://nursingtimes.net/nursing-practice-clinicalresearch/primary-care/ (Accessed: May 21, 2010).

Paillard-Borg, S., Wang, H., Winblad, B. \& Fratiglioni, L. (2009). Pattern of participation in leisure activities among older people in relation to their health conditions and contextual factors: A survey in a Swedish urban area. Ageing and Society 29(5): 803-821.

Reilly, S., Abendstern, M., Huges, J., Challis, V. \& Pedersen, I. (2006). Quality in Long-term care homes for people with dementia: An assessment of specialist provision. Ageing and Society 26(4): 649-668.

Roper, N., Logan, W. W. \& Tierney, A. J. (2000). The Roper-Logan-Tierney Model of Nursing: Based on Activities of Living. Edinburgh: Elsevier Health Sciences.

Rowe, J. W. \& Kahn, R. L. (1997). Successful Aging. The Gerontologist 37: 433-440. 
International Journal of Ageing and Later Life

Townsend, P. (1962). The Last Refuge: A Survey of Residential Institutions and Homes for the Aged in England and Wales. London: Routledge and Kegan Paul.

Ungerson, C. (2000). Thinking about the production and consumption of long-term care in Britain: Does gender still matter. Journal of Social Policy 29(4): 623-643.

Warner Schaie, K., Boron, J. B. \& Willis, S. L. (2005). Everyday Competence in older adults. In M. Johnson, V. L. Bengson, P. Coleman \& T. B. L. Kirkwood (eds.), The Cambridge Handbook of Age and Ageing (pp. 216-228). Cambridge: Cambridge University Press.

WHO (2002). Active Ageing a Policy Framework. Geneva, World Health Organisation Non Communicable Disease Prevention and Health Promotion Organisation. 